Copyright(C2006 IEEE. Reprinted from:

F. Gregorio, S. Werner, J. Cousseau and T.I. Laakso. Channel estimation for multiuser OFDM systems in the presence of power amplifier nonlinearities, in Proceedings of the International Symposium on Personal Indoor and Mobile Radio Communication, PIMRC 2006, September 2006, Helsinki, Finland.

This material is posted here with permission of the IEEE. Such permission of the IEEE does not in any way imply IEEE endorsement of any of Helsinki University of Technology's products or services. Internal or personal use of this material is permitted. However, permission to reprint/republish this material for advertising or promotional purposes or for creating new collective works for resale or redistribution must be obtained from the IEEE by writing to pubs-permissions@ieee.org.

By choosing to view this document, you agree to all provisions of the copyright laws protecting it. 


\title{
ITERATIVE CHANNEL ESTIMATION FOR MULTIUSER OFDM SYSTEMS IN THE PRESENCE OF POWER AMPLIFIER NONLINEARITIES
}

\author{
Fernando H. Gregorio $^{1 *}$ Stefan Werner $^{1} \quad$ Juan E. Cousseau $^{2 \dagger}$ Timo I. Laakso $^{1 \ddagger}$ \\ ${ }^{1}$ Helsinki University of Technology, Signal Processing Laboratory, $\quad{ }^{2}$ Universidad Nacional del Sur \\ P.O. Box 3000, FIN-02015 TKK, Finland. \\ fernando.gregorio@tkk.fi \\ Dept. of Electrical and Computer Eng. \\ Av. Alem 1253, 8000, Bahia Blanca, Argentina
}

\section{ABSTRACT}

This paper proposes a new channel estimation approach for MIMO-OFDM systems subject to power amplifier nonlinearities. The harmful effect of nonlinear distortion on the system performance can be reduced by applying an iterative distortion cancellation technique. However, such an approach requires accurate channel estimates to yield good performance. In our approach, initial channel estimation is carried out in frequency domain (FD) followed by time domain (TD) processing of the received signal. In the TD step, the equalized signals from the FD processing is used to remove the nonlinear distortion and to improve the initial channel estimate. Simulations verify that the combined channel estimation and nonlinear cancellation strategy has a performance close to the case of perfectly known channel. Furthermore, a substantial improvement in the system performance is achieved with the proposed technique when compared with conventional methods in terms of mean square error and BER.

\section{INTRODUCTION}

Multiuser OFDM structures provides high spectral efficiency and increase the system capacity of mobile communication systems [1]. OFDM in combination with multiple antennas enables high transmission rate, specially in the field of wireless local area networks (WLAN).

In space division multiple access (SDMA) different users, each using a single antenna element, are separated at the basestation by the use of multiple antennas. User separation is performed with the aid of user-specific spatial signatures, which are constituted by the channel coefficients between the user antenna and the base station receiver antennas [2]. As a consequence, accurate channel estimates are required to achieve good receiver performance for MIMO-OFDM systems [3] [4].

Power amplifier (PA) nonlinearities have significant impact on the channel estimation process [5]. One option suitable for quasi-stationary channels (e.g., WLAN) is to allocate a group of equally spaced subcarriers to each user. In this way, the peak-to-average power ratio (PAPR) of the OFDM symbol can be reduced as all users transmit training symbols on non-overlapping subcarriers. As a consequence, the power am-

\footnotetext{
* Supported by the Programme AL $\beta$ AN, European Union Programme of High Level Scholarships for Latin America. Identification Number:E03D19254AR. Also supported by Nokia Foundation

${ }^{\dagger}$ This work was partially supported by Universidad Nacional del Sur, Argentina, Project \#24/K023.

$\ddagger$ Fernando Gregorio, Stefan Werner and Timo Laakso are with the Smart and Novel Radios Research Group (SMARAD) Center of Excellence.
}

plifier will mostly operate in the linear region. The channel estimation process is performed once during initialization, and the acquired estimates are used for the rest of the transmission.

This paper will consider the more challenging problem of time-varying channels where the channel needs to be tracked. In such scenario, part of the available subcarriers can be dedicated for pilot symbols, while the rest are employed for data transmission. This leads to large levels of multiuser interference and nonlinear distortion due to an increased PAPR. A power amplifier cancellation technique (PANC) was proposed in [10] that reproduces the PA induced nonlinear distortion and cancel it from the received time-domain signal. After the cancellation of the nonlinear distortion in the time domain (TD), equalization is carried out in frequency domain (FD). Therefore, accurate channel estimates are needed both for the equalization and the nonlinear cancellation steps.

The channel estimation approach proposed here incorporates the channel estimation into the FD and TD loop associated with the PANC technique. The proposed method performs an initial estimate of the channel in FD. The FD channel estimate is refined by performing a second channel estimate in TD. The TD channel estimation is performed after that the nonlinear distortion and multiuser interference have been canceled.

The rest of the paper is organized as follows. Section II introduces the system model. Section III reviews FD and TD channel estimation techniques. The combination of FD and TD channel estimation with the PANC technique is presented in Section IV. Simulation results are provided in Section V, followed by conclusions in Section VI.

In this paper, boldface letters denote vectors and boldface capital letters denote matrices. Furthermore, regular font is used for time-domain variables and calligraphic font is used for frequency-domain variables.

\section{SYSTEM MODEL}

The SDMA-OFDM system under consideration consists of one base station equipped with $P$ antennas and $L$ mobile users with a single transmit antenna, that results in a $P \times L$ MIMO system with $N$ subcarriers. A block diagram of the system is shown in Figure 1.

The transmitted signal from user $j$ at time instant $n$, is given by

$$
\mathbf{x}_{c p_{j}}(n)=\mathbf{G}_{c p} \mathbf{x}_{j}(n)=\mathbf{G}_{c p} \mathbf{Q}_{N} \boldsymbol{X}_{j}(n)
$$

where $\mathbf{G}_{c p}$ is the $(N+v) \times N$ cyclic prefix insertion matrix [6], $v$ is the length of the cyclic prefix, $N+v$ is the total length of the OFDM symbol, $\mathbf{x}_{j}(n)$ is the IDFT of the modulated symbols $\boldsymbol{\chi}_{j}(n) \in \mathbb{C}^{N}$, and $\mathbf{Q}_{N}$ is the $N \times N$ IDFT matrix. 
The multicarrier signal after passing the nonlinear power amplifier consists of two terms

$$
\mathbf{x}_{g_{j}}(n)=K_{L} \mathbf{x}_{c p_{j}}(n)+\mathbf{d}\left[\boldsymbol{x}_{j}(n), g\right]
$$

where the first term $\mathbf{x}_{c p_{j}}(n)$ is the distortion-free discrete input signal vector (time domain) of Eq. (1) and $K_{L}$ is the gain of the linear part. The second term $\mathbf{d}\left[\boldsymbol{x}_{j}(n), g\right]$ is the nonlinear distortion which is a function of the modulated symbol vector $\chi_{j}(n)$ and the power amplifier transfer function $g$.

The received signal at antenna $i$ after cyclic prefix removal, $\mathbf{y}_{i}(n)$, is constituted by the superposition of independently faded signals associated with the $L$ users sharing the same space-frequency resource. The received signal is assumed to be corrupted by Gaussian noise at the array elements and it is given by

$$
\begin{aligned}
\mathbf{y}_{i}(n) & =\sum_{j=1}^{L} \mathbf{H}_{i, j}(n)\left(K_{L} \mathbf{x}_{c p_{j}}(n)+\mathbf{d}\left[\boldsymbol{x}_{j}(n), g\right]\right) \\
& +\mathbf{n}_{i}(n)
\end{aligned}
$$

where $\mathbf{H}_{i, j}(n)$ is an $N \times N$ circulant channel TD matrix response at time instant $n$ formed by the channel response vector $\mathbf{h}_{i, j}$ for the link between user $j$ and base station antenna $i$.

The frequency domain expression of the received signal is obtained by taking the DFT of Eq. (3).

Let $\boldsymbol{y}(n, k)=\left[y_{1}(n, k), \ldots, y_{p}(n, k)\right]^{T}$ denote the vector of received signals at each antenna on subcarrier $k$. Then, the received signal vector for each subcarrier can be written as

$\boldsymbol{y}(n, k)=K_{L} \boldsymbol{H}(n, k) \boldsymbol{x}(n, k)+\boldsymbol{H}(n, k) \boldsymbol{d}(n, k)+\boldsymbol{n}(n, k)$

where $\mathcal{H}(n, k) \in \mathbb{C}^{P \times L}$ is the channel transfer matrix, $\boldsymbol{\chi}(n, k)=\left[\chi_{1}(n, k), \ldots, \chi_{L}(n, k)\right]^{T}$ is the vector containing transmitted signals to each user, $\boldsymbol{d}(n, k)=$ $\left[d_{1}(n, k), \ldots, d_{L}(n, k)\right]^{T}$ are the values of the nonlinear distortion of each user on subcarrier $k, \boldsymbol{n} \in \mathbb{C}^{1 \times P}$ is the additive noise assumed to be circular complex Gaussian with $E\left\{\boldsymbol{n}^{\mathrm{H}} \boldsymbol{n}\right\}=\sigma_{\boldsymbol{n}}^{2} \mathbf{I}$.

The frequency-domain channel transfer matrix $\mathcal{H}(n, k)$ in Eq. (4) is given by

$$
\boldsymbol{\mathcal { H }}(n, k)=\left[\begin{array}{ccc}
\boldsymbol{f}_{1,1}(n, k) & \ldots & \boldsymbol{f}_{1, P}(n, k) \\
\boldsymbol{f}_{2,1}(n, k) & \ldots & \boldsymbol{f}_{2, P}(n, k) \\
\vdots & \ddots & \vdots \\
\boldsymbol{h}_{L, 1}(n, k) & \ldots & \boldsymbol{h}_{L, P}(n, k)
\end{array}\right]
$$

where $h_{i, j}(n, k)$ denotes the channel response on subcarrier $k$ at time $n$ between antenna element $i$ of the base station and user $j$.

\section{A. Frequency-domain equalizers}

The signals transmitted by different users on subcarrier $k$ can be estimated with the aid of a suitable linear combiner $\boldsymbol{W}$, ,resulting in $\widehat{\boldsymbol{X}}(n, k)=\boldsymbol{W}^{\mathrm{H}} \boldsymbol{y}(n, k)$. The frequently used leastsquares (LS) combiner $\boldsymbol{W}_{L S}$ is given by

$$
\boldsymbol{W}_{L S}=\left[\mathcal{H}^{\mathrm{H}}(n, k) \mathcal{H}(n, k)\right]^{-1} \boldsymbol{\mathcal { H }}^{\mathrm{H}}(n, k)
$$

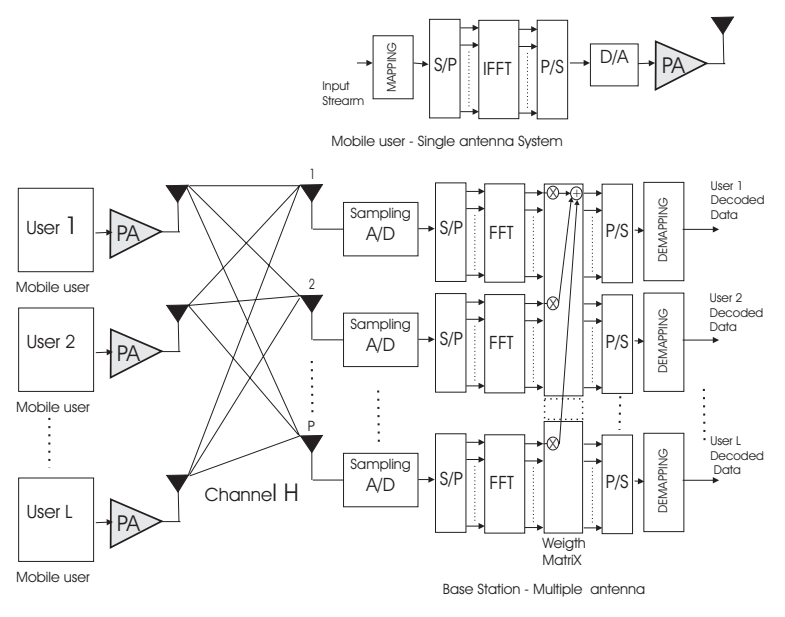

Figure 1: SDMA-OFDM block diagram

Alternatively, a minimum mean-square error (MMSE) detector can be used which exploits the available statistical knowledge and makes a tradeoff between the multiuser interference and the system noise.

\section{ChanNEL ESTIMATION TECHNiQUES}

\section{A. Frequency-domain (FD) channel estimation}

For the frequency domain channel estimation, we will assume a comb-type pilot arrangement where each user is given a set of $T$ dedicated pilot carriers [8]. That is, a total of $T L$ dedicated subcarriers need to be reserved for pilot data, resulting in an overhead $O=T L / N$.

Let $\mathcal{T}_{j}\left(k_{j, 1}, \cdots, k_{j, T}\right)$ denote the set specifying the $T$ pilot carriers of user $j$. The channel frequency response on these subcarriers $\left(k \in \mathcal{T}_{j}\right)$ may be estimated as

$$
\begin{aligned}
\hat{h}_{i, j}(n, k) & =y_{i}(n, k) / \chi_{j}(n, k) \\
& =\digamma_{i, j}(n, k) K_{L}+\hbar_{i, j}(n, k) \frac{d_{j}(n, k)}{\chi_{j}(n, k)}+\frac{n_{i}(n, k)}{\chi_{j}(n, k)}
\end{aligned}
$$

where $y_{i}(n, k)$ is the received signal in the receive antenna $i$ at subcarrier $k \in \mathcal{T}_{L}$ and $\mathcal{X}_{j}(n, k)$ is the training symbol transmitted by user $j$ at the corresponding subcarrier.

Collecting the channel estimates obtained on the pilot carriers for user $j$ into vector $\boldsymbol{h}_{i, j}^{c}(n)=$ $\left[\hat{h}_{i, j}\left(n, k_{j, 1}\right), \cdots, \hat{f}_{i, j}\left(n, k_{j, T}\right)\right]^{T}$, the whole channel FD response can be obtained through interpolation using truncated DFT matrices [7]

$$
\hat{\boldsymbol{h}}_{i, j}(n)=\mathbf{Q}_{N}\left[\mathbf{Q}_{\mathcal{T}_{j}}^{\mathrm{H}} \mathbf{Q}_{\mathcal{T}_{j}}\right]^{-1} \mathbf{Q}_{\mathcal{T}_{j}}^{\mathrm{H}} \hat{\boldsymbol{h}}_{i, j}^{c}(n)
$$

where $\mathbf{Q}_{N}$ is the $N \times N$ IDFT matrix and $\mathbf{Q}_{\mathcal{T}_{j}}$ is the $T$ columns of $\mathbf{Q}_{N}$ associated with the initially estimated subcarriers and the $L_{h}$ rows associated with the nonzero time-domain channel taps.

For the FD channel estimation method described above to work properly, the nonlinear distortion term in Equation (5) needs to be small. Otherwise, the mean-square error of the channel estimates may increase substantially [5]. This problem is addressed with the PANC technique, which is briefly described in Section IV. 


\section{B. Combined frequency domain and time domain channel es- timation}

A combination of FD and TD channel estimation may give a significant improvement in the quality of estimated channel [8]. The idea of a combined FD and TD method is to refine the FD channel estimate in TD in order to reduce the interpolation errors. The TD channel estimation step uses the recovered symbols obtained in the FD process.

First, an initial channel estimate is obtained using the FD estimator, Eq. (6). The initial FD channel estimate is then used to construct the weight matrix $\boldsymbol{W}_{L S}$ for the detection of each user as

$$
\hat{\boldsymbol{\chi}}(n, k)=\boldsymbol{W}_{L S}^{\mathrm{H}} \boldsymbol{y}(n, k)
$$

With the detected symbols, the multiuser interference (MUI) is regenerated and subtracted from the TD input received signal. Thereafter, the channel is estimated in TD using

$$
\hat{\mathbf{h}}_{i, j}(n)=\left[\mathbf{C}^{\mathrm{H}}(n) \mathbf{C}(n)\right]^{-1} \mathbf{C}^{\mathrm{H}}(n) \overline{\mathbf{y}}_{i}(n)
$$

where $\mathbf{C}$ is the $N \times L$ circulant matrix formed by the TD transmitted vector $\mathbf{x}_{j}$, i.e., each column of $\mathbf{C}$ equals the previous column rotated downwards by one element [9], and $\overline{\mathbf{y}}_{i}(n)$ is the TD received signal at antenna $i$ transmitted by user $j$ free of MUI and nonlinear distortion obtained as

$$
\overline{\mathbf{y}}_{i}(n)=\mathbf{y}_{i}(n)-\mathbf{Q}_{N} \sum_{m=1 ; i \neq m}^{L} \operatorname{diag}\left[\hat{\boldsymbol{h}}_{i, m}(n)\right] \hat{\boldsymbol{\chi}}_{m}(n)
$$

In Equation (9), $\operatorname{diag}\left[\hat{\boldsymbol{h}}_{i, m}(n)\right]$ is a diagonal matrix that contains the FD channel estimates between user $m$ and receive antenna $i, \hat{\boldsymbol{X}}_{m}$ contain the recovered symbols and $\mathbf{y}_{i}$ is the time domain received signal at the antenna array $i$ given by Eq. (3).

Finally, the FD channel coefficients are obtained from $\mathcal{H}_{i, j}=\mathbf{Q}_{L}^{\mathrm{H}} \mathbf{h}_{i, j}$ where $\mathbf{Q}_{L}^{\mathrm{H}}$ are the first $L_{h}$ columns of the DFT matrix $\mathbf{Q}_{N}$ ( $L_{h}$ being the number of nonzero channel coefficient in TD). With the new channel estimate, a new weight matrix is calculated and the detection process is carried out with the updated weight matrix. A new iteration may be performed in TD in order to improve the channel estimation accuracy.

\section{FD-TD CHANNEL ESTIMATION WITH NONLINEARITY CANCELLATION TECHNIQUE}

This section considers the combination of channel estimation and the recently proposed power amplifier nonlinearity cancellation (PANC) technique [10].

We start by describing how to estimate the PA model that is used in the PANC stage. Thereafter, a brief review of the PANC technique. Finally, we present the new channel estimator approach that incorporate the PANC into the estimation step to improve the performance of the SDMA-OFDM system.

\section{A. Modeling of power amplifier}

In this paper we assume a solid state power amplifier (SSPA) model. The AM/AM conversion of the SSPA model is given by

$$
g[x(t)]=\frac{|x(t)|}{\left[1+(|x(t)| / A)^{2 p}\right]^{1 / 2 p}}
$$

with the parameter $p$ adjusting the smoothness of the transition from the linear region to the saturation region. The AM/PM conversion is not considered in this PA model. In practice, the SSPA model can be modeled using a memoryless polynomial that can be written as

$$
\hat{g}[x(t)]=\sum_{j=0}^{P} a_{j}|x(t)|^{j}
$$

where $x(t)$ is the input signal, $y(t)$ is the output signal of the power amplifier, and $\left\{a_{j}\right\}_{j=0}^{P}$ are the polynomial coefficients, $P$ being the polynomial order. By exciting the PA with a power-swept single-tone signal, an LS estimate of the coefficients $a_{j}$ can be obtained, see [11] for details. We stress the PANC technique can be easily implemented with more complicated PA models (e.g., including memory effects). However, this is beyond the scope of the paper.

\section{B. Power Amplifier Nonlinearity Cancellation Technique} The distortion term $d_{j}(n, k)$ in Equation (5) affects the channel estimation process. However, with a good knowledge of the PA model as discussed in the previous section, the receiver can estimate $\hat{d}_{j}(n, k)$ from the received signal $y_{i}(n, k)$, and thereafter remove it. The PANC technique, first uses an initial estimate of $\hat{\chi}_{j}(n, k)$ to estimate the distortion $\hat{d}_{j}(n, k)$. The distortion is then removed from the original received signal and an improved estimate of $\chi_{j}(n, k)$ can be obtained. This second estimation $\hat{\chi}_{j}(n, k)$ can be used to re-estimate the distortion vector. The process can be performed iteratively.

The PANC technique can be summarized as ${ }^{1}$ :

At iteration $m$

1) Estimate symbols $\hat{\chi}_{j}^{m}(n, k)=\left\langle\frac{y_{i}(n, k)}{\hbar_{i, j}(n, k)}-\hat{d}_{j}(n, k)\right\rangle$

2) Temporal domain $\hat{\mathbf{x}}_{j}^{m}(n)=\mathbf{Q} \hat{\boldsymbol{X}}_{j}^{m}(n)$

3) Estimate distortion term $\hat{\mathbf{d}}_{j}(n)=g\left[\hat{\mathbf{x}}_{j}^{m}(n)\right]-\hat{\mathbf{x}}_{j}^{m}(n)$

4) Distortion in frequency domain $\hat{\boldsymbol{d}}_{j}(n)=\mathbf{Q}^{\mathrm{H}} \hat{\mathbf{d}}_{j}(n)$

5) New iteration

Note that at iteration $m=0$, the distortion term is set to zero. The new distortion terms are picked from vector $\hat{\boldsymbol{d}}_{j}(n)=\left[\hat{\boldsymbol{d}}_{j}(n, 1) \cdots, \hat{\boldsymbol{d}}_{j}(n, N)\right]^{\mathrm{T}}$. For more details on the PANC technique, see [10].

\section{Frequency and time domain (FD-TD) channel estimation with PANC}

The iterative channel estimation technique that combines FDTD channel estimation with PANC can be summarized in two fundamental steps:

\section{Step 1 - FD channel estimation with PANC}

Each user signal is initially detected using the channel estimate obtained from the previous OFDM symbol, i.e., using $\hat{\boldsymbol{h}}_{i, j}(n \mid n-1)$. The initial symbol estimates are used to get a first estimate of the nonlinear distortion term, as discussed in previous subsection. Thereafter, a new channel estimation is

\footnotetext{
${ }^{1}\langle$,$\rangle denotes the symbol decision process in which the decoder chooses the$ value that is closest in Euclidean distance to $\hat{\chi}_{j}(n, k)$
} 


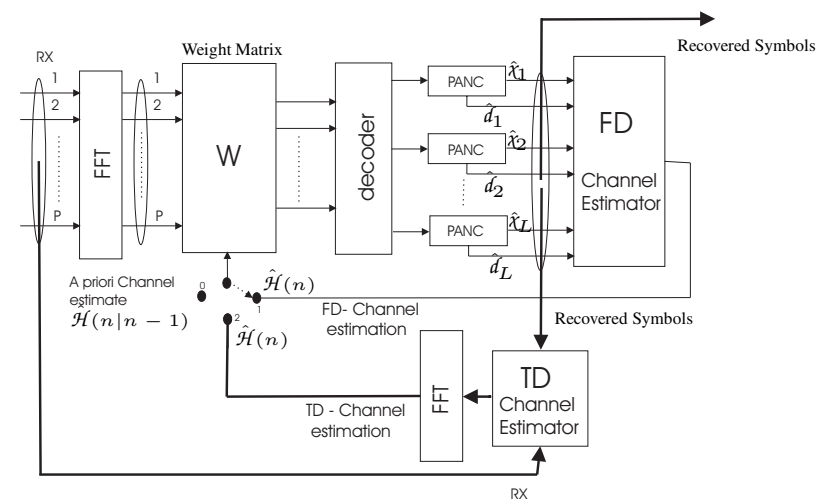

Figure 2: FD-TD Channel estimation

carried out on the pilot carriers which includes the nonlinear distortion:

$$
\hat{\boldsymbol{h}}_{i, j}(n, k)=\frac{y_{i}(n, k)}{\chi_{j}(n, k)+\hat{d}_{j}(n, k)} .
$$

The complete channel frequency response, $\hat{\boldsymbol{h}}_{i, j}(n)$ between user $j$ and receive antenna $i$ is obtained with Eq. (6). With the more up-to-date FD channel estimate, each user signal can be detected using the LS detector.

\section{Step 2 - TD channel estimation with PANC}

In the second step, TD channel estimation is employed. With the detected symbols from previous step, the nonlinear distortion and the multiuser interference are calculated and their effect is subtracted from the time-domain received signal

$\overline{\mathbf{y}}_{i}(n)=\mathbf{y}_{i}(n)-\mathbf{Q}_{N} \sum_{m=1 ; j \neq m}^{L} \operatorname{diag}\left[\hat{\boldsymbol{h}}_{i, m}(n)\right]\left[\hat{\boldsymbol{x}}_{m}(n)+\hat{\boldsymbol{d}}_{m}(n)\right]$

The time domain channel response is given by Eq. (8). A block diagram of the FD-TD channel estimation with PANC is illustrated in Figure 2.

\section{Simulation RESUlts}

The performance of the PANC technique with FD-TD channel estimation (CE) was evaluated in an SDMA-OFDM system with $N=512$ subcarriers, $P=4$ receive antennas in the base station, and $L=4$ users with a single transmit antenna.The carrier frequency is $f_{c}=2.4 \mathrm{GHz}$. A four-path Rayleigh fading channel with independent propagation paths is implemented. The paths are generated using Jake's Doppler spectrum. Power loss of the channel taps are $[0,-1,-3,-9] \mathrm{dB}$ with the delay profiles equal to $[0,1,2,3] \mu s$, that corresponds to a typical urban scenario. The speeds of the $L$ mobile users were set to $30 \mathrm{~km} / \mathrm{h}$. The number of pilot subcarriers per user was set to $T=16$. The MSE for FD-TD and FD channel estimation for a system with an SSPA power amplifier with clipping level $\gamma=\frac{A}{\sqrt{E_{n}\left\{|x(n)|^{2}\right\}}}=1 \mathrm{~dB}$ and smoothness factor $p=2$ and QPSK constellation is shown in Figure 3. The MSE for the cases of linear and nonlinear amplifiers are also included to evaluate the improvement achieved with PANC combined with an FD-TD channel estimation approach. For comparison, the
MSE achieved when only using an FD channel estimation is included. As can be seen from the figure, the combination of PANC and FD-TD results in MSE levels that approach those obtained with a linear amplifier.

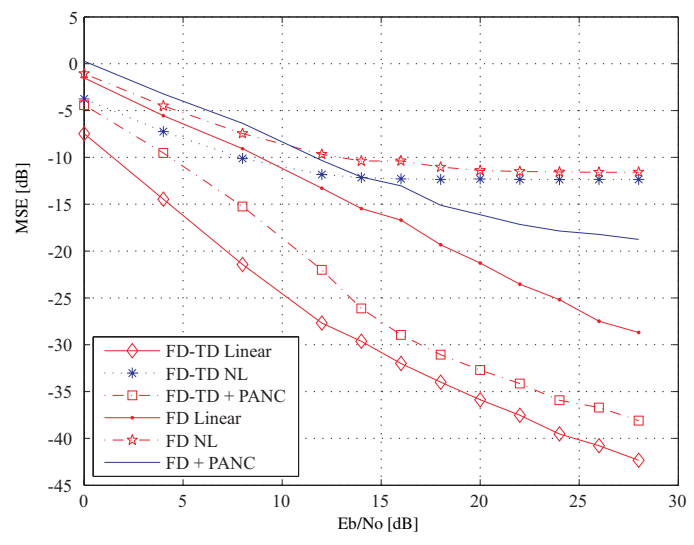

(a) SDMA-OFDM P $=4, \mathrm{~L}=2$

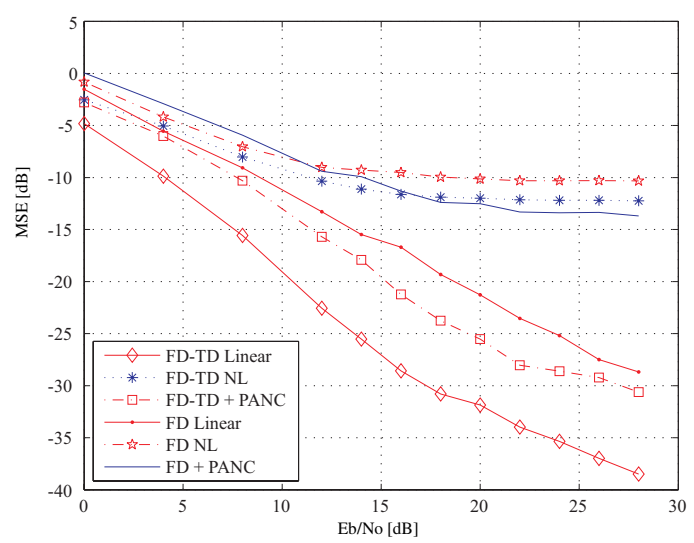

(b) SDMA-OFDM $\mathrm{P}=4, \mathrm{~L}=4$

Figure 3: QPSK - MSE of FD-TD and FD channel estimation

In order to compare the performance of the novel technique with other methods that combat the PA nonlinearity effects, the predistorter (PD) in [11] was implemented. The PD was modeled with a 5 th order polynomial for a realistic SSPA model with $\gamma=4 \mathrm{~dB}$ and $p=2$.

Figure 4 shows MSE channel estimation curves for a PD system employing FD-TD channel estimation and the system using PANC for $P=4$ and $L=2$. The transmitted symbols were taken from a 16-QAM constellation. The curves for linear amplifier and nonlinear (NL) amplifier cases are included. In order to show the robustness of the channel estimation technique to PA modeling errors, we also included the results obtained when using a 7th order polynomial PA model in the receiver.

Also, the combination of PD and PANC was evaluated. The results do not show a significant improvement on the BER values. However, this combination can be useful because the predistorter provides a reduction in the out-of-band distortion and PANC is useful for the in-band region.

The curves presented shows the advantage of using the PANC technique compared with a solution using a PD. The 
PANC outperforms the PD in MSE with approximately $20 \mathrm{~dB}$ at high SNR.

BER simulation results are also shown in Figure 5. An SNR gain larger than $2 \mathrm{~dB}$ is obtained with PANC when compared with $\mathrm{PD}$ for $L=1$ user. A large SNR gain is also obtained for the case of $L=2$.

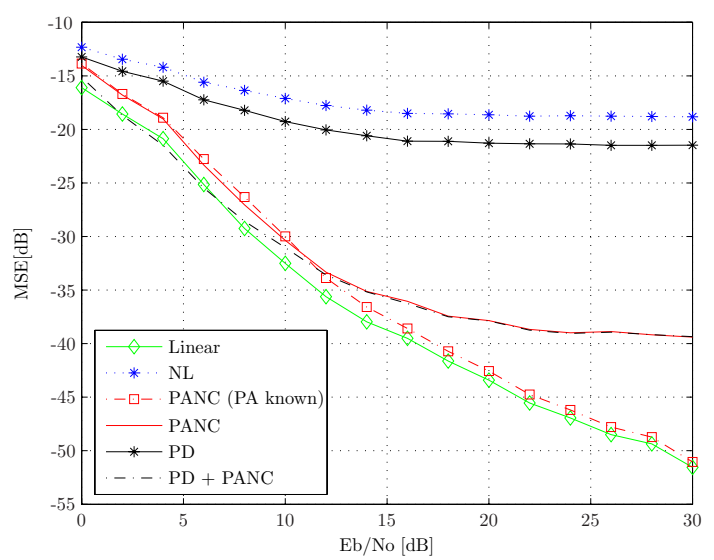

(a) SDMA-OFDM $P=4, L=1$

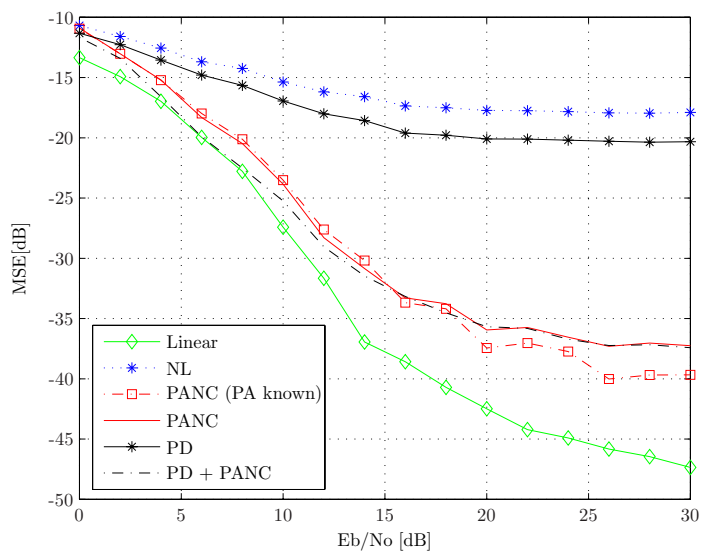

(b) SDMA-OFDM $P=4, L=2$

Figure 4: 16-QAM - MSE of FD-TD channel estimation

\section{CONCLUSIONS}

This paper presented a new channel estimation structure for MIMO-OFDM systems that can be used when reducing nonlinear power amplifier (PA) effects. The new approach incorporates the channel estimation into the PA nonlinearity cancellation technique, and channel estimation is performed in both frequency and time domains.

It was verified that when incorporating the new channel estimation strategy into the PA nonlinearity cancellation technique, the performance was close to that of perfectly known channel. Thus, preserving the significant improvement in BER levels that can be obtained when compared with a solution that ignores the nonlinear PA effects.

Simulations showed that the PA nonlinearity cancellation structure outperforms a system using a predistorter. The performance of a solution that combines the nonlinear cancellation technique with a predistorter solution was also evaluated, which was verified to yield reduced out-of-band distortion and improved BER levels.

The robustness of the technique to power amplifier modelling errors was also verified.

\section{REFERENCES}

[1] P. Vandenameele, Space division multiple access for wireless local area networks, Kluwer, 2001.

[2] L. Hanzo, M. Munster, B. J. Choi, and T. Keller, OFDM and MC-CDMA for broadband multi-user communication, WLANs and broadcasting, Wiley, 2003.

[3] H. Miao and M. Juntti, "Space-time channel estimation and performance analysis for wireless MIMO-OFDM systems with spatial correlation, " IEEE Trans. Veh. Technol., vol. 54, no. 6, pp. 2003-2016, Nov. 2005

[4] Y. Li, "Simplified channel estimation for OFDM systems with multiple transmit antennas, "IEEE Trans. Comm., vol. 1, no. 1, pp. 67-75, Jan. 2002

[5] B. Wu, S. Cheng, and H. Wang, "Clipping effects on channel estimation and signal detection in OFDM," in PIMRC 2003, Sept. 2003, vol. 1, pp. $531-534$.

[6] T. Roman, M. Enescu, and V. Koivunen, "Joint time-domain tracking of channel and frequency offsets for MIMO OFDM systems," Kluwer Wireless Personal Commun., vol. 31, pp. 181-200, 2004.

[7] L. Deneire et al., "A low-complexity ML channel estimator for OFDM," IEEE Trans. Commun., vol. 51, pp. 135-140, Feb. 2003.

[8] S. Werner, M. Enescu, and V. Koivunen, "Combined frequency and time domain channel estimation in mobile MIMO-OFDM systems," in IEEE, ICASSP 2006, May 2006.

[9] G. Golub and C. F. V. Loan, Matrix Computations, The Johns Hopkins University Press, 1993.

[10] F. Gregorio, T. Laakso, and J. Cousseau, "Receiver cancellation of nonlinear power amplifier distortion in SDMA-OFDM systems," in IEEE ICASSP 2006, France, May 2006.

[11] Y. Guo and J. Cavallaro, "Enhanced power efficiency of mobile OFDM radio using predistortion and post-compensation," in IEEE VTC 2002Fall., Sept. 2002, vol. 1, pp. $214-218$.

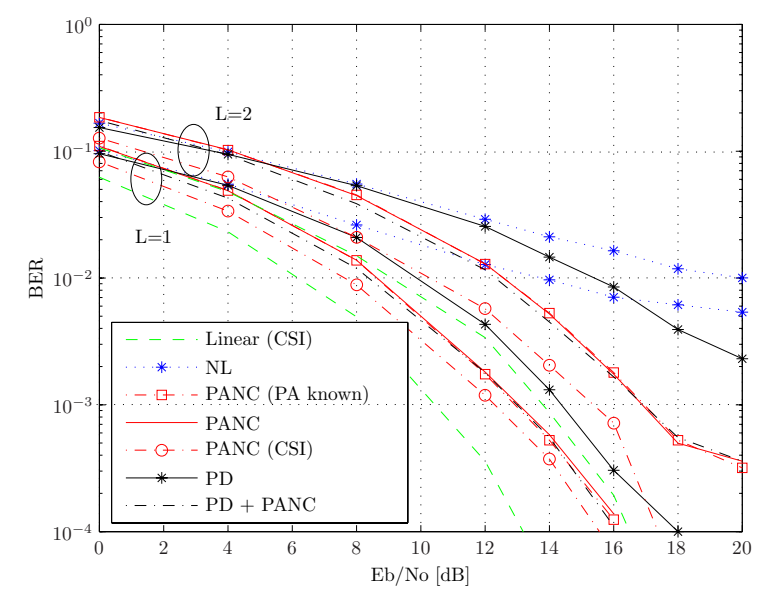

Figure 5: BER - 16-QAM system $P=4$ and $L=2$ with FD-TD channel estimation 